\title{
Antidepressiva bei leichten depressiven Störungen
}

\author{
Antidepressants For Mild Depressive Disorders
}

\section{Bibliografie}

Dol http://dx.doi.org/

10.1055/s-0031-1276865

Psychiat Prax 2011; 38:

270-273

(c) Georg Thieme Verlag KC

Stuttgart · New York .

ISSN 0303-4259

\section{Korrespondenzadressen}

Priv.-Doz. Dr.

Thomas C. Baghai

Klinik für Psychiatrie und Psychotherapie

Ludwig-Maximilians-Universität

Nußbaumstraße 7

80336 München

Baghai@med.uni-muenchen.de

Priv.-Doz. Dr. Tom Bschor

Abteilung für Psychiatrie

Schlosspark-Klinik

Heubnerweg 2

14059 Berlin

bschor@schlosspark-klinik.de

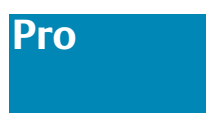

Laut der Weltgesundheitsorganisation (WHO) sind depressive Störungen von herausragender gesundheitsökonomischer Bedeutung und verursachen neben dem Leidensdruck der Betroffenen häufig psychosoziale Beeinträchtigungen und Erwerbsminderung [1].

Eine adäquate Therapie besteht aus einer multimodalen Kombinationstherapie, welche biologische pharmakologische und nichtpharmakologische Therapien, Psychotherapie und Soziotherapie mit individuellem Schwerpunkt, der sich im Behandlungsverlauf verändern kann, einsetzt. Hierbei wird versucht, eine individualisierte Behandlung mit größtmöglicher Sicherheit und Verträglichkeit sowie optimaler Ansprechwahrscheinlichkeit durchzuführen.

Aufgrund ihrer guten Verträglichkeit und der breiten Erfahrung werden selektive Serotoninwiederaufnahmehemmer (SSRI) meist als Mittel der ersten Wahl eingesetzt. Ebenso kommen jedoch Präparate mit dualem Wirkmechanismus wie selektive Serotonin- und Noradrenalinwiederaufnahmehemmer (SNRI), noradrenerg und spezifisch serotonerg (NaSSA) wirkende $\alpha_{2}$-Blocker, ein selektiver Dopamin- und Noradrenalinwiederaufnahmehemmer (DNRI) infrage. Aufgrund der guten Verträglichkeit kommen aber ebenso ein selektiver Noradrenalinwiederaufnahmehemmer (NARI), ein reversibler selektiver Hemmer der Monoaminooxidase A (RIMA) oder ein Melatoninrezeptorenagonist mit $5-\mathrm{HT}_{2} \mathrm{Cre}-$ zeptorenantagonistischen Eigenschaften infrage. Trizyklische Antidepressiva (TCA) werden hingegen seltener, irreversible MAO-Hemmer aus Sicherheitsgründen nur als Therapie der zweiten Wahl bei Pharmakotherapieresistenz eingesetzt [2].

Obwohl in kontrollierten Studien nach Gabe von Antidepressiva Nichtansprechraten um die 30\% festestellt wurden [3] und diese bei naturalistischen Studiendesigns sogar noch höher ausfallen können [4], ist die Effektivität einer antidepressiven Pharmakotherapie bei mittel- bis schwergradigen Depressionen durch kontrollierte klinische Prüfungen gut belegt und in Metaanalysen bestätigt worden [5].

\section{Differenzierung leichtgradiger \\ Depressionen}

Depressive Episoden können anhand ihres Schweregrads in schwere, mäßiggradige und leichte Depressionen eingeteilt werden. Diagnosesysteme wie die Internationale Klassifikation der WHO (ICD-10) [6] gehen rudimentär auf eine Schweregradeinteilung ein, spezifische Ratingskalen wie z.B. die Hamilton- (Hamilton rating scale for depression, HAM-D) [7] oder die Montgomery-Åsberg-Skala (Montgomery-Åsberg depression rating scale, MADRS) [8] sind jedoch besser geeignet. Die Sensitivität dieser Skalen für Veränderungen der depressiven Symptomatik gerade bei leichtgradigen Depressionen wird jedoch als suboptimal beurteilt [9].

Von besonderer klinischer Bedeutung ist die Schweregradeinteilung, weil Leitlinien wie z.B. die des National Institute for Clinical Excellence (NICE) [10] eine medikamentöse Behandlung primär nur für mittelgradige bis schwere, nicht aber für leichtgradige Depressionen empfehlen und sich die S3-Leitlinien der Deutschen Gesellschaft für Psychiatrie, Psychotherapie und Nervenheilkunde teilweise an diesen Empfehlungen orientieren [11]. Für die Empfehlung, bei milderen Formen depressiver Erkrankungen zunächst nur psychotherapeutische Interventionen einzusetzen, Antidepressiva nur bei Therapieresistenz gegenüber diesen Verfahren zuzulassen [10] und sich lediglich mit einer Beobachtung des Krankheitsverlaufs („active monitoring“ [12]) zu begnügen, gibt es jedoch derzeit noch keine ausreichende empirische Grundlage, daher wird sie weiterhin kontrovers diskutiert. Für die Notwendigkeit der Behandlung von mittelschweren bis schwergradi- 
gen Depressionen mit Antidepressiva, häufig mit psycho- und soziotherapeutischer Begleitung, besteht jedoch ein breiter klinischer und wissenschaftlicher Konsens in allen wichtigen Leitlinien (z.B. World Federation of Societies of Biological Psychiatry, WFSBP [13], American Psychiatric Association, APA [14]).

\section{Therapie leichtgradiger Depressionen}

Da für die klinische Wirksamkeit verschiedenster antidepressiver Therapiestrategien eine gute Evidenzlage besteht, werden in den gängigen Leitlinien unterschiedliche Ansätze präzisiert. Die bereits erwähnte aktive Beobachtung [12] sollte zunächst neben der Berücksichtigung psychosozialer Probleme auch Psychoedukation mit weitergehenden Informationen über depressive Erkrankungen beinhalten. Auch die DGPPN empfiehlt im Falle von leichten depressiven Episoden eine aktiv abwartende Begleitung [11] für zunächst 2 Wochen, da möglicherweise die Symptomatik auch ohne aktive Behandlung abklingt. Allerdings wird auch in diesen Leitlinien darauf hingewiesen, dass nach kritischer Nutzen- / Risikoabwägung Antidepressiva auch bei leichten depressiven Episoden eingesetzt werden können. Eine ähnliche Empfehlung kann man den amerikanischen Leitlinien der APA [14] sowie den Leitlinien der WFSBP [13] entnehmen.

In einer Metaanalyse wurde über eine verminderte Wirksamkeit antidepressiver Pharmakotherapien bei leichtgradigen Depressionen berichtet [15], allerdings konnten inzwischen methodische Mängel dieser Untersuchung belegt werden [16], die eine sichere Interpretation ausschließen.

Der therapeutische Nutzen einer Pharmakotherapie bei leichten Depressionen ist ausreichend gut belegt. Selbst Präparate, für die es keinen Wirksamkeitsnachweis bei schweren Depressionen gibt, sind in der Behandlung leichtgradiger Depressionen wirksam. Dies gilt z.B. für Johanniskraut/Hypericum perforatum $[17,18]$, das trotz eines nicht unbeträchtlichen Interaktionspotenzials sehr gut verträglich ist, oder für Benzodiazepine [19], deren langfristiger Einsatz bei depressiven Störungen aufgrund des Toleranz- und Abhängigkeitspotenzials kontraindiziert ist. Selektive Serotoninwiederaufnahmehemmer werden aus Verträglichkeitsgründen häufig als Mittel der ersten Wahl zur Behandlung depressiver Störungen eingesetzt. Ein Wirksamkeitsnachweis bei leichtgradigen Depressionen wurde in dieser Gruppe beispielsweise für Fluoxetin [20] oder Sertralin [21] erbracht. Gleiches wurde in der Gruppe dual wirksamer selektiver Serotoninund Noradrenalinwiederaufnahmehemmer für Duloxetin belegt [22]. Das trizyklische Antidepressivum Amitriptylin war bei leichtgradigen Depressionen mit einem HAM-D17-Summenscore $\geq 13$ ebenfalls wirksam, allerdings konnte bei noch geringerem Schweregrad < 13 keine ausreichende Wirksamkeit gezeigt werden $[23,24]$. Die Behandlung leichtgradiger depressiver Erkrankungen im Rahmen einer antidepressiven Pharmakotherapie kann daher generell empfohlen werden [2,25], dies gilt insbesondere für Patienten, die eine frühzeitige Symptombesserung innerhalb der ersten beiden Behandlungswochen aufweisen [26]. Es gibt einen Konsens darüber, dass auch leichtgradige Depressionen von einer antidepressiven Pharmakotherapie profitieren können [2] und es einer individuellen Nutzen- / Risikoanalyse bedarf, die auch die Risiken der Unterlassung einer effektiven Therapie mit der Möglichkeit der Entwicklung depressiver Erkrankungen schwererer und chronischer Ausprägung in die Überlegungen einbezieht.

\section{Therapie subsyndromaler Depressionen}

Subsyndromale Depressionen erfüllen die diagnostischen Kriterien von ICD-10 oder DSM-IV nicht vollständig, können aber trotzdem behandlungsbedürftig sein. Mit ihnen assoziierte funktionelle Defizite können bei subsyndromaler oder unterschwelliger Depression zu massiven psychosozialen Beeinträchtigungen führen $[27,28]$ und sogar einer Beeinträchtigung gleichen, wie sie das Vollbild einer depressiven Erkrankung hervorruft [29]. Zudem besteht ein nicht unerhebliches Risiko der Befundverschlechterung bis hin zum Vollbild einer depressiven Episode oder rezidivierenden depressiven Störung [30] sowie zur Entwicklung einer sich chronisch verschlechternden Erkrankung [31].

Bisher angewandte Untersuchungsdesigns in randomisierten kontrollierten Studien waren unglücklicherweise nur unzureichend geeignet, um die Besserung subsyndromaler Depressionen durch Antidepressiva ausreichend zu belegen [32], ihre bessere Berücksichtigung in zukünftigen Studien wurde daher gefordert [29]. Gut belegt ist jedoch, dass vor allem die Persistenz subsyndromaler depressiver Symptome nach inkompletter Remission einer depressiven Episode eine Langzeittherapie mit Antidepressiva erfordert um die Symptomatik zu verbessern und einen erneuten Rückfall einer schwereren Depression zu verhindern [33]. Aus Sicht des Klinikers ist daher die konsequente Behandlung subsyndromaler Depressionen angezeigt und wird trotz der noch unzureichenden Evidenzlage speziell dann empfohlen, wenn besondere Risikofaktoren wie z. B. eine positive Familienanamnese für Depressionen, Suizidalität, fortbestehende Residualsymptomatik nach Teilremission einer schwereren Depression oder eine außergewöhnliche psychosoziale Belastungssituation vorliegen [28].

\section{Fazit}

Eine antidepressive Therapie besteht heutzutage aus einem Therapieangebot, das pharmako-, psycho- und soziotherapeutische Elemente mit individuellen Schwerpunkten enthält. Diese werden durch psychiatrische und somatische klinische Faktoren, aber natürlich auch durch die Patientenpräferenz mitbestimmt. Während bei mittel- und schwergradigen Depressionen die Mitanwendung biologischer Therapieverfahren (meist Pharmakotherapie) obligat ist, muss bei leichtgradigen Depressionen im Sinne einer partizipativen Entscheidungsfindung neben dem individuellen Risiko, eine schwerergradige oder chronifizierte Depression mit all ihren psychiatrischen und somatischen Risiken zu entwickeln, auch die Entscheidung der betroffenen Patienten berücksichtigt werden. Bei erhöhten Risiken kann daher eher zu einer begleitenden antidepressiven Pharmakotherapie geraten werden, falls diese fehlen, kann die in vielen Leitlinien vorgeschlagene aktive Beobachtung zumindest vorübergehend ausreichend sein. Unabhängig davon sind die aktive Begleitung betroffener Patienten und die Koordination sowie gegebenenfalls auch die Verlagerung der psychiatrischen Therapieschwerpunkte obligatorischer Bestandteil einer lege artis durchgeführten Therapie.

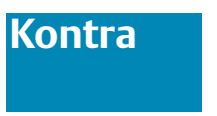

Die Neuentwicklung selektiver Antidepressiva in den letzten 20 Jahren hat nicht zu einer $\mathrm{Zu}$ nahme der Wirksamkeit geführt, sondern vor allem die Verträglichkeit der Medikamente verbessert. Auch bei der ambulant am häufigsten verordneten Antidepressivagruppe, den SSRI, handelt es sich jedoch keineswegs um unbedenkliche Medikamente. Zum Beispiel findet sich der von den SSRI ge- 
hemmte Serotonintransporter auch auf Thrombozyten, was die Wahrscheinlichkeit gastrointestinaler Blutungen erhöhen kann [34]. Auch die relativ häufigen Hyponatriämien sind ernsthafte Komplikationen. Bei jungen (erwachsenen) Patienten ist zudem eine Zunahme des Suizidrisikos durch SSRI wahrscheinlich [35]. Allein aus diesen Gründen muss die Indikation für eine Behandlung eindeutig sein. Bedeutung gewinnen diese Risiken auch angesichts der Tatsache, dass im Praxisalltag in Deutschland wahrscheinlich eine große Anzahl von Patienten Antidepressiva erhalten, obwohl sie noch nicht einmal die Kriterien einer depressiven Episode erfüllen [36].

Erste Forderung zur Stellung einer eindeutigen Behandlungsindikation ist die nachgewiesene Wirksamkeit der Therapie. Da schon lange bekannt ist, dass die Wirksamkeit einer Antidepressivatherapie (in Abgrenzung zu Placebo) mit dem Schweregrad der Depression ansteigt, ist die große Aufmerksamkeit, die die Metaanalyse von Zulassungsstudien (eingereicht bei der USamerikanischen Zulassungsbehörde FDA) von Kirsch et al. 2008 [15] erzeugt hat, nicht ganz nachvollziehbar. Wie vielfach diskutiert, hatte die Analyse ergeben, dass bei leichten Depressionen praktisch kein Wirksamkeitsunterschied zwischen Antidepressiva und Placebo besteht und erst bei sehr schweren Depressionen von einem klinisch relevanten Unterschied gesprochen werden kann. Höchstrangig in JAMA publiziert, wurde in der Zwischenzeit dieses Ergebnis nochmals bestätigt [37]. Aktuell hat eine doppelblinde, randomisierte Studie erneut gezeigt, dass Citalopram, aber auch das häufig bei leichten Depressionen eingesetzte Johanniskraut, keine über Placebo hinausgehende Wirksamkeit in der Behandlung leichter Depressionen hat, stattdessen eine erhöhte Nebenwirkungsrate besteht [38].

Die fehlende Differenz zwischen Placebo- und Antidepressivawirkung bei leichten Depressionen verwundert nicht, wenn man den insgesamt sehr hohen Anteil des Placeboeffekts an der Besserung einer Depression unter Antidepressivabehandlung betrachtet. Systematische Analysen ergaben, dass dieser zwischen $68 \%$ [39] und 75\% [40] ausmacht. Möglicherweise unterschätzen diese Zahlen sogar noch den tatsächlichen Anteil des Placeboeffekts, da Studien mit aktivem (d.h. typische Nebenwirkungen imitierenden) Placebo aufgrund ethischer Aspekte nicht mehr durchgeführt werden dürfen und zudem negative Studien, d.h. solche, in denen sich Antidepressivum und Placebo nicht unterschieden, systematisch seltener publiziert werden. Wie kürzlich publiziert [41], werden 97\% aller positiven Studien in Fachzeitschriften veröffentlicht, während dieser Anteil für die negativen Studien nur bei 33\% liegt (tatsächlich als negativ publiziert werden sogar nur 12\%). „Positiv“ im Sinne einer signifikanten Überlegenheit des Verumpräparates ist dabei nicht mit einer klinischen Relevanz des Unterschieds gleichzusetzen, da bei hinreichend großen Studien auch Unterschiede von lediglich 1 oder 2 Punkten auf der Hamilton-Depressionsskala für das Erreichen des Signifikanzniveaus ausreichen, während Patienten diesen Unterschied vermutlich überhaupt nicht bemerken.

Eine weitere Sorge sollte uns veranlassen, die Indikation für eine Antidepressivamedikation streng $\mathrm{zu}$ stellen. Immer häufiger wird vermutet, dass der Beginn einer Behandlung den Langzeitverlauf einer depressiven Erkrankung verschlechtern könnte [42]. Es wird befürchtet, dass die pharmakologisch induzierten Veränderungen des zentralnervösen Stoffwechsels letztlich zu einer Zunahme der Vulnerabilität für eine erneute depressive Episode führen, insbesondere wenn nach gegebener Zeit die Antidepressivamedikation wieder beendet wird. Die recht gut belegte Wirkung von Antidepressiva in der Erhaltungstherapie und der Rezidivprophylaxe [43] wäre unter diesem Blickwinkel dann nur der Beleg dafür, dass der Organismus nach dem erfolgten Eingriff in die zentralnervöse Regulation auf das Antidepressivum nicht mehr verzichten kann.

Die unter Federführung der DGPPN vor Kurzem veröffentlichte S3-Leitlinie bzw. Nationale Versorgungsleitlinie Unipolare Depression [44], die gemäß der S3-Methodik im Konsens mit allen relevanten Gruppierungen und unter systematischer Aufarbeitung der gesamten internationalen Literatur (z. B. auch der englischen NICE Guidelines [45]) erstellt wurde, kommt daher zu der ausdrücklichen Empfehlung, „Antidepressiva sollten nicht generell zur Erstbehandlung bei leichten depressiven Episoden eingesetzt werden, sondern allenfalls unter besonders kritischer Abwägung des Nutzen-Risiko-Verhältnisses“. Eine solche Empfehlung, verbunden mit Konzepten der „Stepped Care“ (NICE), ist also gut begründet und sollte auch nicht unter Verweis auf die Gegebenheiten des Praxisalltags ignoriert werden. Dies gilt auch deshalb, weil zur Therapie bei leichten bis mittelschweren Depressionen gute Evidenzen für Psychotherapie (Einzel oder Gruppen) und auch niedrigschwellige psychologische Interventionen (Beratung, Problemlösung, Verhaltensaktivierung) möglich sind (Zusammenfassung bei [45]).

Zum Abschluss zurück zum Placeboeffekt: Vom übergroßen Ausmaß des Placeboeffekts in der Depressionsbehandlung [46] war schon die Rede. Wir alle haben Kurven aus klinischen Studien vor Augen, in denen es auch im Placeboarm zu einem eindrucksvollen Rückgang der Depression kommt. Wäre es da nicht ein Fehler, mit dem Verzicht auf ein Antidepressivum auf diesen Effekt zu verzichten?

Zur Beantwortung dieser Frage lohnt es, den Placeboeffekt näher zu betrachten. Wirksam ist dabei vermutlich die Erwartungshaltung der Patienten, verbunden mit ärztlicher/therapeutischer Zuwendung, dem Ernstnehmen des Patienten, der Vermittlung von Hoffnung u.a. durch ein erklärbares Krankheits- und Behandlungskonzept, dem Stellen einer Diagnose und der Entlastung des Patienten durch eine klare Attribuierung der Symptomatik als Erkrankung. Dies sind wichtige, sehr potente und weitgehend nebenwirkungsfreie Wirkfaktoren (nicht nur in der Depressionsbehandlung), die die Heilungskräfte der Patienten mobilisieren. Es wäre aber ein echter Fehlschluss anzunehmen, dass diese Faktoren zwingend an die Verordnung eines Medikaments gekoppelt sind. Unsere Aufgabe, insbesondere in der Behandlung leichter Depressionen, ist es vielmehr, diese Faktoren in guter medizinischer Tradition durch ärztlich-menschliche $\mathrm{Zu}$ wendung und Empathie sowie durch psychotherapeutische Begleitung unserer Patienten zur Entfaltung zu bringen.

\section{Literatur}

1 World Health Organization. The global burden of disease: 2004 update 2004

2 Sartorius N, Baghai TC, Baldwin DS et al. Antidepressant medications and other treatments of depressive disorders: a CINP Task Force report based on a review of evidence. Int J Neuropsychopharmacol 2007; 10 (Suppl. 1): S1-207

3 Charney DS, Grothe DR, Smith SL et al. Overview of psychiatric disorders and the role of newer antidepressants. J Clin Psychiatry 2002; 63: 3-9

4 Fava M, Rush AJ, Wisniewski SR et al. A comparison of mirtazapine and nortriptyline following two consecutive failed medication treatments for depressed outpatients: a STAR*D report. Am J Psychiatry 2006; 163: $1161-1172$

5 Arroll B, Elley CR, Fishman $T$ et al. Antidepressants versus placebo for depression in primary care. Cochrane Database Syst Rev 2009: CD007954 
6 World Health Organization. The ICD-10 Classification of Mental and Behavioural Disorders: Clinical Descriptions and Diagnostic Guidelines. Geneva, Switzerland: 1992

7 Hamilton M. A rating scale for depression. J Neurol Neurosurg Psychiatry 1960; 23: 56-62

8 Montgomery SA, Asberg M. A new depression scale designed to be sensitive to change. Br J Psychiatry 1979; 134: 382-389

9 Helmreich I, Wagner S, Mergl R et al. The Inventory of Depressive Symptomatology (IDS-C(28)) is more sensitive to changes in depressive symptomatology than the Hamilton Depression Rating Scale (HAMD (17)) in patients with mild major, minor or subsyndromal depression. Eur Arch Psychiatry Clin Neurosci 2010 Dec 4 (Epub ahead of print)

10 National Institute for Health and Clinical Excellence. Depression: The treatment and management of depression in adults. Clinical Guideline 90. http://www.nice.org.uk/nicemedia/live/12329/45888/45888.pdf, 2009

11 DGPPN S3-Leitlinie/Nationale Versorgungs-Leitlinie Unipolare Depression. 2009

12 National Collaborating Centre for Mental Health. Depression in Adults (update); Depression: the treatment and management of depression in adults. London: National Institute for Health and Clinical Excellence (NICE); 2009

13 Bauer M, Bschor T, Pfennig A et al. World Federation of Societies of Biological Psychiatry (WFSBP) Guidelines for Biological Treatment of Unipolar Depressive Disorders in Primary Care. World J Biol Psychiatry 2007; 8: 67-104

14 Karasu TB, Gelenberg A, Merriam A et al. Practice guideline for the treatment of patients with major depressive disorder (revision). American Psychiatric Association. Am J Psychiatry 2000; 157: 1-45

15 Kirsch I, Deacon BJ, Huedo-Medina TB et al. Initial severity and antidepressant benefits: a meta-analysis of data submitted to the Food and Drug Administration. PloS Med 2008; 5: e45

16 Fountoulakis KN, Möller HJ. Efficacy of antidepressants: a re-analysis and re-interpretation of the Kirsch data. Int J Neuropsychopharmacol 2011; 14 : 405-412

17 Kasper S, Gastpar M, Muller WE et al. Efficacy of St. John's wort extract WS 5570 in acute treatment of mild depression: a reanalysis of data from controlled clinical trials. Eur Arch Psychiatry Clin Neurosci 2008; 258: 59-63

18 Laakmann G, Schüle C, Baghai TC et al. St. John's wort in mild to moderate depression: the relevance of hyperforin for the clinical efficacy. Pharmacopsychiatry 1998; 31 (Suppl. 1): 54-59

19 Laakmann G, Faltermaier-Temizel M, Bossert-Zaudig $S$ et al. Are benzodiazepines antidepressants? Psychopharmacology (Berl) 1996; 124: 291-292

20 Volz HP, Laux P. Potential treatment for subthreshold and mild depression: a comparison of St. John's wort extracts and fluoxetine. Compr Psychiatry 2000; 41: 133-137

21 Hegerl U, Hautzinger M, Mergl R et al. Effects of pharmacotherapy and psychotherapy in depressed primary-care patients: a randomized, controlled trial including a patients' choice arm. Int J Neuropsychopharmacol 2010; 13: 31-44

22 Shelton $\mathrm{RC}$, Andorn AC, Mallinckrodt $\mathrm{CH}$ et al. Evidence for the efficacy of duloxetine in treating mild, moderate, and severe depression. Int Clin Psychopharmacol 2007; 22: 348-355

23 Paykel ES, Freeling P, Hollyman JA. Are tricyclic antidepressants useful for mild depression? A placebo controlled trial. Pharmacopsychiatry 1988; 21: 15-18

24 Paykel ES, Hollyman JA, Freeling P et al. Predictors of therapeutic benefit from amitriptyline in mild depression: a general practice placebocontrolled trial. J Affect Disord 1988; 14: 83-95

25 Hegerl U, Pfeiffer-Gerschel T, Althaus D. [Elements for the effective treatment of patients with depressive disorders at the primary care level]. MMW Fortschr Med 2004; 146 Spec No 2: 10, 12-10, 14
26 Tadic A, Helmreich I, Mergl R et al. Early improvement is a predictor of treatment outcome in patients with mild major, minor or subsyndromal depression. J Affect Disord 2010; 120: 86-93

27 Altshuler LL, Post RM, Black DO et al. Subsyndromal depressive symptoms are associated with functional impairment in patients with bipolar disorder: results of a large, multisite study. J Clin Psychiatry 2006; 67: $1551-1560$

28 Hegerl $U$, Schönknecht $P$. [Subdiagnostic depression. Are there treatments with clinically relevant effects?]. Nervenarzt 2009; 80: 532-539

29 Marangell LB, Dennehy EB, Miyahara S et al. The functional impact of subsyndromal depressive symptoms in bipolar disorder: data from STEP-BD. J Affect Disord 2009; 114: 58-67

30 Lyness JM. Naturalistic outcomes of minor and subsyndromal depression in older primary care patients. Int J Geriatr Psychiatry 2008; 23: 773-781

31 Lyness JM, Chapman BP, McGriff J et al. One-year outcomes of minor and subsyndromal depression in older primary care patients. Int Psychogeriatr 2009; 21: 60-68

32 Gelenberg AJ, Thase ME, Meyer RE et al. The history and current state of antidepressant clinical trial design: a call to action for proof-of-concept studies. J Clin Psychiatry 2008; 69: 1513-1528

33 Nierenberg AA. Diagnosing and treating patients with symptoms of depression. J Clin Psychiatry 2009; 70: e05

34 Strubel T, Birkhofer A, Mossmer $G$ et al. Blutungsrisiko unter SSRI-Behandlung. Nervenarzt 2010; 81: 549-555

35 Stone M, Laughren T, Jones Ml et al. Risk of suicidality in clinical trials of antidepressants in adults: analysis of proprietary data submitted to US Food and Drug Administration. BMJ 2009; 339: b2880

36 Ghaemi SN. Why antidepressants are not antidepressants: STEP-BD, $\mathrm{STAR}^{*} \mathrm{D}$, and the return of neurotic depression. Bipolar Disord 2008 10: 957-968

37 Fournier JC, Derubeis RJ, Hollon SD et al. Antidepressant drug effects and depression severity: a patient-level meta-analysis. JAMA 2010; 303: 47-53

38 Rapaport M, Nierenberg A, Howland R et al. The treatment of minor depression with St. John's Wort or citalopram: Failure to show benefit over placebo. J Psychiatr Res 2011; 45: 931-941

39 Rief $W$, Nestoriuc $Y$, Weiss S et al. Meta-analysis of the placebo response in antidepressant trials. J Affect Disord 2009; 118: 1-8

40 Kirsch I, Sapirstein G. Listening to Prozac but Hearing Placebo: A MetaAnalysis of Antidepressant Medication. Prevention \& Treatment 1998; 1 : Article 0002a

41 Turner E, Matthews A, Linardatos E et al. Selective publication of antidepressant trials and its influence on apparent efficacy. N Engl J Med 2008; 358: 252-260

42 Fava G. Can long-term treatment with antidepressant drugs worsen the course of depression? J Clin Psychiatry 2003; 64: 123-133

43 Geddes JR, Carney SM, Davies C et al. Relapse prevention with antidepressant drug treatment in depressive disorders: a systematic review. Lancet 2003; 361: 653-661

44 DGPPN, BÄK, KBV, AWMF, AkdÄ, BPtK, DAGSHG, DEGAM, DGPM, DGPS, DGRW Hrsg, für die Leitliniengruppe Unipolare Depression. S3-Leitlinie / Nationale VersorgungsLeitlinie Unipolare Depression - Langfassung. Berlin, Düsseldorf: DGPPN, ÄZQ AWMF; 2009

45 National Collaborating Centre for Mental Health, National Institute for Clinical Excellence (NICE). Depression: Management of depression in primary and secondary care. Clinical Guideline 23. www.nice.org.uk/ page.aspx?o=235213, 2004

46 Walsh BT, Seidman SN, Sysko R et al. Placebo response in studies of major depression: variable, substantial, and growing. JAMA 2002; 287: 1840-1847 